\title{
Étude de cas: les débuts de la stérilisation légale des malades mentaux dans le canton de Vaud*
}

Jacques Gasser et Geneviève Heller

\section{Summary}

The topic of genetic transmission of mental diseases appears early in the psychiatric literature. Among the consequences of these theories in the Swiss canton Vaud a law was enacted in 1928 concerning the sterilization of persons affected by «mental disease or mental disability». This short communication presents a selection of case histories which sheds light on the first years after the enactment of this law.

\section{Résumé}

La question de la transmission héréditaire des maladies mentales est présente tôt dans les textes des aliénistes. Dans le canton de Vaud, une des conséquences de ces théories a été l'élaboration en 1928 d'un article de loi concernant la stérilisation des personnes atteintes «de maladie mentale ou d'une infirmité mentale». Dans cette brève communication, nous présentons quelques cas concrets qui illustrent les premières années de l'application de cette loi.

\footnotetext{
*Cet article présente la première étape d'une recherche effectuée dans le cadre de l'Institut universitaire d'histoire de la médecine et de la santé publique et mandatée par les Hospices et le Service de la Santé publique du Canton de Vaud. La deuxième étape de la recherche, confiée à Gilles Jeanmonod, portera sur toute la période de la stérilisation légale des malades mentaux dans le canton de Vaud, soit de 1929 à 1985. Un projet de recherche portant sur les autres cantons romands est envisagé.
}

Dr Jacques Gasser et Geneviève Heller, Institut universitaire d'histoire de la médecine et de la santé publique, Case postale 196,1000 Lausanne 4 


\section{Introduction}

Depuis la fin du mois d'août 1997, une nouvelle polémique a atteint la Suisse à propos de sa politique durant la première moitié de notre siècle. En effet, suite à deux articles du quotidien suédois Dagens Nyheter, révélant la stérilisation «eugénique» forcée de plus de 60000 personnes entre 1935 et 1976 en Suède ${ }^{1}$, la presse s'est interrogée sur la situation de la Suisse. Dans ce cadre le rôle du canton de Vaud, pionnier en la matière, a de nouveau été mis en avant par sa fameuse loi du 3 septembre 1928 qui introduit un nouvel article 28 bis dans la loi de 1901 sur le régime des personnes atteintes de maladies mentales; il établit les conditions dans lesquelles la stérilisation légale et coercitive des malades mentaux est autorisée. Après l'affaire des fonds juifs en déshérence et de l'or nazi, la Suisse est ainsi à nouveau confrontée à son passé.

En tant qu'historiens de la psychiatrie, nous sommes interpellés par ces dernières révélations et par les simplifications et les amalgames qu'on a pu lire dans la presse quotidienne lors de la révélation des événements suédois. Ainsi, s'il serait intéressant de s'interroger sur le rôle que la société demande aux historiens de jouer dans l'urgence de l'actualité et durant les prochaines années à l'intérieur des différentes commissions d'enquête qui se mettent en place, nous nous contenterons d'exposer ici quelques faits préliminaires qui devraient permettre de nourrir le débat.

Notre étude des stérilisations légales des malades mentaux porte dans un premier temps uniquement sur la situation du canton de Vaud lors des années qui ont suivi la mise en place de la nouvelle loi et elle bénéficie de la consultation des archives du Service de la Santé publique et de ceux de l'Hôpital psychiatrique universitaire du canton de Vaud.

La question de la transmission héréditaire des maladies mentales est présente très tôt dans les textes des aliénistes. Elle a été étudiée notamment par Claude Bénichou² et Ian Dowbiggin ${ }^{3}$.

Dans les dernières années du XIX ${ }^{\mathrm{e}}$ siècle, la question de l'hérédité en psychiatrie est utilisée pour donner une explication organique de la maladie mentale alors qu'avec l'eugénisme, qui se développe dans le premier tiers du

1 La recherche à la base de ces articles a été effectuée par Maija Runcis, chercheur au département d'histoire à l'Université de Stockholm, qui prépare une thèse sur la stérilisation forcée en Suède.

2 Claude Benichou (textes réunis et présentés par), L'ordre des caractères. Aspects de l'hérédité dans l'histoire des sciences de l'homme, Paris: Vrin, 1989.

3 Ian Dowbiggin, La folie héréditaire ou comment la psychiatrie française s'est constituée en un corps de savoir et de pouvoir dans la seconde moitié du XIX'e, Paris: E.P.E.L., 1993. 
$\mathrm{XX}^{\mathrm{e}}$ siècle, on tente d'apporter des solutions pratiques à l'hypothétique hérédité morbide.

Une des conséquences du rôle prépondérant accordé à l'hérédité dans les théories psychiatriques et du développement des théories de la dégénérescence et de l'eugénisme est, dans le cas particulier du canton de Vaud, la légalisation de mesures contraignantes ayant pour but de limiter les effets néfastes de l'hérédité des maladies mentales.

\section{L'article 28 bis de 1928}

La loi du 14 février 1901 sur le régime des personnes atteintes de maladies mentales ne contient aucune allusion à des possibilités de limiter la transmission héréditaire des maladies mentales. C'est le 3 septembre 1928 que le législatif vaudois ajoute un nouvel article 28 bis à la loi de 1901. En voici l'intitulé:

«Une personne atteinte de maladie mentale ou d'une infirmité mentale peut être l'objet de mesures d'ordre médical pour empêcher la survenance d'enfants, si elle est reconnue incurable et si, selon toutes prévisions, elle ne peut avoir qu'une descendance tarée. L'intervention médicale n'a lieu que sur autorisation du Conseil de santé. Le Conseil de santé lui-même ne donne cette autorisation qu'après enquête et sur préavis conforme de deux médecins désignés par lui. Il décide de l'attribution des frais.» ${ }^{4}$

Cet article de loi est le premier en Europe à légiférer sur cette question délicate bien que la pratique de stérilisation des aliénés ait été déjà effective depuis la fin du XIX ${ }^{\mathrm{e}}$ siècle à Zurich ${ }^{5}$ et au moins depuis 1919 dans le canton de Vaud 6 .

Les premières lois sur la stérilisation sont d'origine américaine ${ }^{7}$. Entre 1907 et 1930,29 États, soit près des deux tiers des États-Unis, ont adopté une loi qui vise principalement les malades mentaux et les criminels placés dans des institutions d'État.

4 Recueil des lois, décrets, arrêtés et autres actes du gouvernement du canton de Vaud, 1928, p. 70.

5 S. Frank, Praktische Erfahrungen mit Kastration und Sterilisation psychisch Defekter in der Schweiz, Berlin: Karger, 1925.

$6 \mathrm{H}$. Steck, «La pratique de la stérilisation légale des anormaux psychiques dans le canton de Vaud», Revue médicale de la Suisse romande, 55, 1935, p. 874-895; R. Bersier, Contribution à l'étude de la liberté personnelle. L'internement des aliénés et des asociaux. La stérilisation des aliénés, Cremines: Roos, 1968.

7 R. Penel, «La stérilisation eugénique en Amérique», Hygiène mentale. L'Informateur des Aliénistes et des Neurologistes, 25 (7), 1930, p. 173-188. 
Le projet de l'article 28 bis a suscité des discussions nourries sur un sujet considéré comme «grave». Beaucoup de «perplexité», une «angoisse morale» ont entouré les débats durant lesquels les arguments des opposants ont été largement exprimés ${ }^{8}$.

L'article 28 bis est cependant accepté, il se cantonne au domaine en principe exclusif de la maladie ou de l'infirmité mentale. «L'incapable doit être protégé contre toute intervention hâtive ou intéressée et cette protection doit être organisée par la loi.» ${ }^{9}$

En juillet 1930, soit un peu plus d'un an après l'entrée en vigueur de l'article 28 bis, le chef du Service sanitaire cantonal, F. Wanner, publie un article ${ }^{10}$ dans le périodique français Hygiène mentale, expliquant que seize demandes ont été adressées au Conseil de santé pour une population d'environ 320000 habitants. Neuf demandes ont été repoussées ${ }^{11}$, sept autorisations ont été accordées et finalement six stérilisations ont été effectuées. A titre de comparaison, on a pu repérer 18 demandes durant l'année $1933^{12}$ dont les dossiers ont été conservés dans les archives du Conseil de santé. On dénombre six refus, six autorisations, six demandes abandonnées provisoirement ou non.

\section{Deux cas d'autorisation de stérilisation}

Parmi les sept autorisations de la première année présentées par Wanner, quatre concernent des «idiotes» ou «faibles d'esprit», trois des femmes atteintes de maladie mentale.

On retiendra à titre d'exemple le cas de «[Jeanne $]^{13}, 19$ ans, imbécillité».

8 Bulletin des séances du Grand Conseil du Canton de Vaud, [BGC] séance du 22 mai 1928, p. 507-539 et du 3 septembre 1928, p. 897-916.

9 BGC, 22 mai 1928 , p. 515.

$10 \mathrm{~F}$. Wanner, «La loi sur la stérilisation des personnes privées de discernement et son fonctionnement dans le canton de Vaud pendant la première année», Hygiène mentale. L'Informateur des Aliénistes et des Neurologistes, 25 (7),1930, p. 163-172.

11 Une étude a porté sur le contexte de cette loi et sur son application. On doit relever que l'auteur n'a pas pris en considération les cas refusés par le Conseil de santé et qu'il minimise le rôle protecteur de la loi (Philippe Ehrenström, La stérilisation des malades mentaux et l'avortement eugénique dans le canton de Vaud. Eugénisme et question sociale, mémoire de la Faculté des Lettres de Genève, 1989; plusieurs articles du même auteur ont été publiés, notamment «Eugénisme et santé publique : la stérilisation légale des malades mentaux dans le canton de Vaud (Suisse)», History and Philosophy of the Life Sciences, 15, 1993, p. 205-227).

12 Archives cantonales vaudoises (ACV), Fonds K VIII non répertorié, classeur no. 327, 1932-1934.

13 Les prénoms donnés ici sont fictifs. 
«Fille illégitime, [Jeanne] a été élevée dans un milieu déplorable; a reçu l'instruction de l'école primaire, mais sans pouvoir suivre d'une façon satisfaisante; ne peut répondre qu'aux questions qui sont habituellement résolues par des enfants de 9 ans. Placée dans un institut de l'Armée du Salut, elle est déclarée au bout de plusieurs mois incapable de tout développement; il faudra, dit la direction de cet institut, la placer dans un asile pour idiots. Inhabile aux travaux manuels, elle est incapable d'élever des enfants; aux termes du Code civil, le mariage ne peut être autorisé. Physiquement assez bien développée avec un léger goitre. Hérédité: père inconnu; mère faible d'esprit; a deux soeurs mariées, un peu plus intelligentes, et un frère qui l'est peu; la grand-mère qui a élevé [Jeanne] est débile de corps et d'esprit, paresseuse et sans ordre; dans une seule chambre vivent la grand-mère, la tante, un jeune homme et la jeune [Jeanne]. Un frère de [Jeanne] est également arriéré. La stérilisation est autorisée et l'intervention exécutée.» ${ }^{14}$

Les dossiers de cette première année n'ont pas été retrouvés dans les archives du Conseil de santé. Par contre, le dossier médical d'une personne admise à l'asile d'aliénés est conservé dans les archives de Cery. C'est le deuxième cas retenu ici, celui de «[Germaine], 28 ans, schizophrénie».

«N'a présenté des symptômes d'altération psychique qu'à l'âge de 22 ans; mariée à 21 ans, elle divorce au bout d'une année et présente à ce moment une période d'agitation bizarre. Nouvelle poussée d'agitation en 1926, après laquelle elle peut reprendre ses occupations. En 1928, elle doit être internée; au bout d'une année on peut songer à la laisser sortir de l'Asile, mais son caractère reste fermé, instable, elle est érotique et son père demande la stérilisation. Dans l'hérédité de Mme [...], nous ne retrouvons qu'un oncle maternel bizarre, fuyant la société, incapable de travailler. Sa façon de se conduire fait penser à la schizophrénie. L'autorisation est accordée et la malade opérée.» ${ }^{15}$

Cette présentation est un résumé des données du dossier de Cery.

Ce dernier indique pourquoi la jeune fille est amenée à Cery par son père. Elle a traversé à deux reprises, quatre ans et deux ans auparavant, des états critiques:

«La malade avait un rire bizarre, elle ne parlait presque pas, disait voir des bêtes, entendait des voix, [...] avait l'habitude de boucher les trous et les fentes dans sa chambre, [...] était anxieuse, $[\ldots]$ ne travaillait plus, restait au lit [...].»

Ses parents la soignèrent chez eux plusieurs mois. Depuis un mois, elle est revenue chez elle, avec les mêmes symptômes, mais:

«Hier au soir elle tomba sur sa mère, lui déchira ses habits sans raisons, voulait s'échapper. Se mit à courir dans la rue. Nuit sans sommeil. Allait tout le temps de son lit à la porte. Médecin appelé à minuit.»16

Les commentaires, durant le séjour de la jeune fille à l'asile, alternent selon les journées:

«A refusé de se lever, se tortille dans son lit et rit aux éclats. [...] Depuis quinze jours, se lève régulièrement, s'occupe bien. [...] Grogne comme un porc des quarts d'heure durant de façon stéréotypée. [...] Demande à me parler: depuis quinze jours [...] elle se sent toute changée. Elle voudrait être libre. [...]»

14 Wanner, op. cit., p. 170-171.

15 Wanner, op. cit., p. 172.

16 Le certificat médical requis pour l'admission n'a pas été retrouvé dans le dossier. 
Le lendemain de ce dernier commentaire du 20 décembre, le médecin écrit au père une lettre dont nous n'avons pas connaissance; le 7 janvier, le père écrit:

«En réponse à votre communication du 21 décembre écoulé, par laquelle vous m’informez que ma fille [...] désire avec insistance sa sortie et qu'elle ne peut plus supporter d'être restreinte dans sa liberté, ce dont je vous remercie, je crois devoir vous dire que la situation de ma fille n'est plus en état de servir la «société〉 et par-dessus le marché «femme aux mœurs légères〉. Craignant des naissances illégitimes, j’ai pris la résolution de faire une demande à l'autorité compétente en vue de lui faire subir la stérilisation. En conséquence, je me permets de vous demander comment je dois m'y prendre et dans quelle mesure vous pourriez y collaborer. En attendant une résolution de tout cela, il me semble que c'est tout indiqué de prolonger son internement, ce ne peut être que pour son bien!»

Après avoir reçu cette lettre, le médecin parle à la patiente de la volonte du père. «Elle accueille cette proposition très tranquillement, mais elle dit ne pas bien en comprendre la nécessité. Elle croit qu'elle n'est plus normale, qu'elle n'a plus d'ovaires. [...] Veut encore réfléchir.»

Un mois plus tard, «dit qu'elle a réfléchi et qu'elle n'a rien contre, désire que cela se fasse le plus vite possible. Elle ne veut pas rester indéfiniment ici pour cette raison.»

On doit remarquer dans cet exemple que la stérilisation est envisagée comme une alternative à l'internement comme cela a été maintes fois évoqué lors de la discussion de l'article 28 bis au sein du Grand Conseil.

Quelques jours après, le médecin de Cery envoie son rapport au Service sanitaire en y joignant la lettre du père. Il reprend les éléments du dossier qui l'amènent au diagnostic de schizophrénie au sujet de la jeune femme, il mentionne en outre le consentement de celle-ci à la stérilisation, consentement accordé en échange d'une promesse de liberté. La patiente traverse une période de rémission permettant d'envisager sa sortie sous certaines conditions.

«L'enfant qui naîtrait d'elle aurait grande chance de tomber malade à son tour. [...] Selon les statistiques les plus récentes [...] [l'enfant serait] schizophrène [...] [dans] 8 à $9 \%$ [des cas], [...] [présenterait un] caractère anormal [...] [dans] 40 à $45 \%$ [des cas]. [...] Il faut donc dire que plus de la moitié des enfants d'un schizophrène seraient tarés. [...] Il est certain qu'elle court le risque de devenir enceinte à cause de son érotisme. Sa mentalité spéciale la rend incapable d'élever un enfant, de sorte que cet enfant, déjà chargé au point de vue héréditaire manquerait [...] des soins dont il aurait encore plus besoin que d'autres. Il aurait double malchance d'être taré constitutionnellement et de manquer d'un milieu familial favorable.»

La préoccupation du médecin, nourrie par ses hypothèses relatives à l'hérédité, est renforcée par le handicap éducatif que représente la maladie de la mère.

Le médecin de Cery évoque ensuite l'évolution des traitements des aliénés qui favorise une mise en liberté précoce:

«Cet avantage a ses mauvais côtés, puisqu'il permet justement à ces malades de procréer des enfants qui tomberont à leur tour malades. Plusieurs psychiatres allemands ont attiré dernièrement l'attention du public médical sur cet inconvénient et ils demandent l'application 
de mesures prophylactiques contre cette mauvaise sélection, telles qu'elles sont prévues justement par la nouvelle loi vaudoise.»

Dans le dossier de Cery, on trouve la réponse du chef du Service sanitaire, le Dr Wanner, envoyée quatre mois après que la demande a été présentée:

«L'enquête prévue par la loi a été ordonnée et le rapport des experts, soumis au Conseil de santé, lequel ne voit pas d'opposition à ce que la stérilisation soit effectuée. Nous vous prions de bien vouloir demander le transfert de votre fille de l'Asile de Cery à la Maternité.»

Après la stérilisation, elle a pu retourner chez ses parents, mais quinze jours plus tard, elle est ramenée par son frère et sa sœur avec une lettre du père qui demande la réadmission de sa fille à Cery. Son état s'est de nouveau aggravé et «sa mère s'en effraie à chaque instant». Son deuxième séjour à l'asile va durer quinze ans au cours duquel elle passe par des périodes de crise et d'autres de rémission.

La stérilisation avait été demandée pour permettre la sortie de l'asile psychiatrique d'une schizophrène dont on craignait les effets de la maladie sur sa descendance. Le diagnostic s'est confirmé par la suite.

\section{Deux cas de refus de stérilisation}

Les neufs demandes de stérilisation, formulées le plus souvent par les communes, puis refusées par le Conseil de santé durant l'année 1929, ont pour motifs l'alcoolisme, le vagabondage, l'incapacité d'une personne à gagner sa vie, le désordre, les grossesses illégitimes chez des femmes sans ressources. Quant au motif de refus de la part du Conseil de santé, c'est l'absence de maladie mentale ou de faiblesse d'esprit.

Deux dossiers, tirés de l'année 1933, apportent davantage de renseignements sur les motivations des uns et des autres.

Le premier concerne une demande adressée par une municipalité pour que le Conseil de santé «ordonne la stérilisation des époux [...]»: l'homme «dont le casier judiciaire est abondamment pourvu de 12 condamnations» est «paresseux» et «ne fera jamais rien pour sortir du paupérisme intégral dans lequel il se complaît»; quant à sa femme, elle est «une personne d'une fainéantise peu commune», elle tient «des propos orduriers». Ils ont trois enfants, «bientôt quatre [...] débiles, atteints de rachitisme, strabisme, etc.» ${ }^{17}$. La Municipalité ajoute dans sa lettre que «la tradition de cette famille [...] est de vivre aux dépens de la collectivité. [...] Nous estimons le moment venu de

17 On doit relever ici la confusion des pathologies dans l'esprit des autorités locales, comme si toutes ces maladies étaient mentales et héréditaires. 
mettre un frein à l'activité sexuelle des époux [...] en atteignant le mal à sa source et ceci dans l'intérêt de la communauté.» Cette.dernière phrase a attiré l'attention du Conseil de santé: en marge est apposé un point d'exclamation au crayon rouge.

Le préavis du Conseil est défavorable, les motifs sont considérés comme non valables et l'article 28 bis n'est pas applicable. Il faudrait au moins, remarque-t-on, que la demande soit appuyée d'un certificat médical.

La demande, comme à chaque requête, circule par correspondance auprès des membres du Conseil qui donnent leur opinion et signent. L'un d'eux semble particulièrement irrité: «Je croyais qu'il avait été décidé d'adresser une circulaire à toutes les municipalités pour les rendre attentives au sens et à la portée de la loi.» ${ }^{18}$

Un mois après la requête de la municipalité, le chef du Département de l'Intérieur répond négativement. Celle-ci répond à son tour qu'«elle regrette la décision» et qu'elle déplore «bien davantage que la loi sanitaire ne prévoie pas, au même titre que les malades mentaux, la stérilisation des parasites». Elle ajoute d'ailleurs quelques arguments à ceux de sa première lettre, notamment que le mari est tuberculeux et «adepte [...] des théories communistes. [...] Nous persistons à croire que le meilleur moyen de lutter contre le paupérisme reste dans ce cas particulier la stérilisation.» Le Conseil de santé maintient sa décision.

Dans le second dossier, une autre municipalité demande la stérilisation d'une ressortissante de 25 ans, domestique. Celle-ci, de faible santé, ayant un enfant illégitime qui est placé aux frais de l'assistance communale, attend un deuxième enfant illégitime «résultant de sa mauvaise conduite» (ce qui s'avérera erroné, la démarche étant faite sans que la municipalité vérifie ses allégations). Elle est «en outre un peu faible d'esprit». La municipalité de domicile qui a signalé le cas à la municipalité d'origine a précisé qu'elle vit dans un «triste milieu». Le Conseil de santé demande un rapport médical au médecin délégué ${ }^{19}$ de la région où habite la jeune fille.

Ce dernier donne des renseignements très développés, résultant d'une enquête sociale autant que médicale: orpheline de mère dès l'âge de 12 ans, elle a été placée chez un particulier jusqu'à l'âge de 16 ans. Dès lors, soit dès sa sortie de l'école, elle a travaillé. Elle a fait à trois reprises des séjours de

18 Souligné dans le texte.

19 «Les médecins délégués sont les représentants du Département de l'Intérieur auprès des préfets, des autorités communales, des commissions de salubrité et des particuliers», Exposé des motifs de la loi sanitaire, $B G C, 1928$, p. 23. 
plusieurs mois dans une infirmerie (phlébite, maux de tête) ${ }^{20}$. Le médecin délégué précise qu'elle n'est pas enceinte et, de plus, «ne présente pas d'affection pouvant justifier l'application de l'article 28 bis. Elle présente bien peutêtre un léger déficit psychique et une intelligence plutôt modeste, mais pas à un point tel que des mesures spéciales doivent être prises contre elle.»

Le Conseil de santé adresse sa réponse à la municipalité: «Votre demande de stérilisation n'est pas recevable.» Elle transmet aussi la conclusion du rapport du médecin délégué: «Doit être sortie du milieu actuel au plus vite et placée dans une bonne famille où elle soit bien surveillée.»

\section{Conclusion}

Les premières études de cas que nous avons pu effectuer montrent que la loi est certainement protectrice, elle représente un frein à une partie des stérilisations motivées exclusivement ou principalement par la pauvreté, l'alcoolisme, un comportement asocial. C'était bien là le principal propos de cette législation. Ceci n'exclut pourtant pas des stérilisations de personnes démunies, défavorisées, notamment des jeunes filles, souvent orphelines, placées très tôt chez des particuliers, dont la scolarité a été perturbée par le travail imposé, et qui sont à la merci des hommes de leur entourage qui commettent un, voire plusieurs attentats à la pudeur très lourds de conséquences pour elles. Donc une partie des jeunes filles ont été protégées par la loi, pas toutes. Si l'hérédité était chargée, si les facultés mentales semblaient limitées (appréciées dans les années 30 et 40 trop souvent selon des critères scolaires compréhension d'une lecture, calcul, connaissances géographiques, etc.), la débilité mentale risquait fort d'être attestée sans que des affections plus graves puissent être mises en évidence.

La loi pouvait aussi ne pas être prise en compte. Dans ce cas on peut supposer que cela arrivait plutôt chez des particuliers n'étant pas à l'assistance ni sous tutelle, c'est-à-dire sans interférence administrative et financière des diverses autorités. Les stérilisations non soumises à autorisation, que l'on peut qualifier de clandestines ou de privées, devaient concerner de manière prioritaire des femmes de milieu économiquement et socialement moyen, sinon privilégié. Mais celles-ci échappent probablement à toute observation historique et à toute estimation statistique.

20 Le Conseil de santé prolongera l'enquête auprès de l'infirmerie dont les observations précisent qu'elle a souffert de névralgies. Une note, difficile à déchiffrer, est ajoutée, résultant de renseignements oraux: "plaignante sempiternelle, hypocondre». La jeune fille n'a guère trouvé de soulagements à ses maux, ni, semble-t-il, de compréhension. 